\title{
Rationalizing the Formation of Belinostat Solvates with Experimental Screening and Computational Prediction
}

Zhonghua Li a, Ruiling Ouyang a, Peng Shi ${ }^{a}$, Shichao Du ${ }^{a}$, Junbo Gong ${ }^{a, b}$, Songgu $W u^{a^{*}}$

a. State Key Laboratory of Chemical Engineering, School of Chemical Engineering and Technology, Tianjin University, Tianjin 300072, People's Republic of China.

b. Chemistry and Chemical Engineering Guangdong Laboratory, Shantou 515031, People's Republic of China.

Contents of Supporting Information

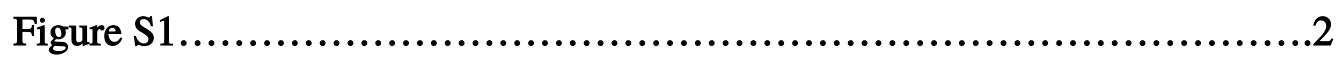

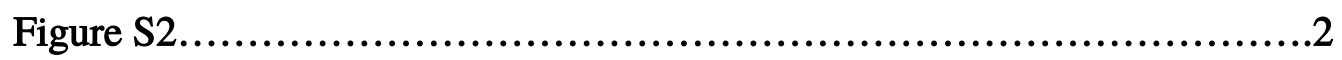

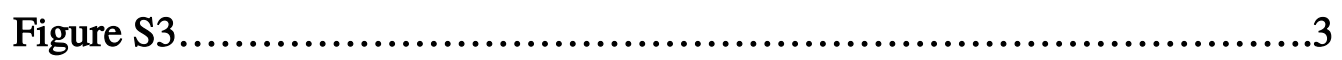

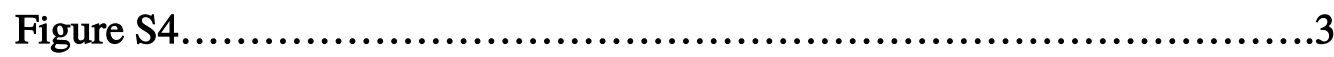

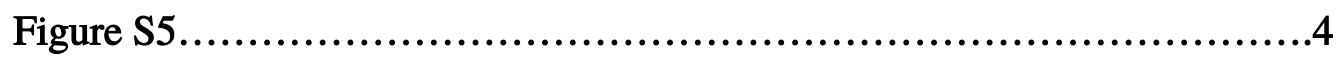

Figure $\$$ (6..........................................................4

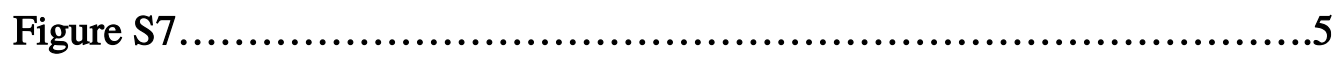

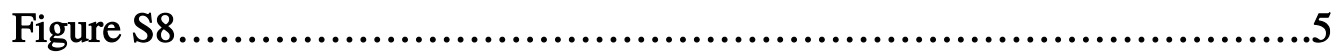

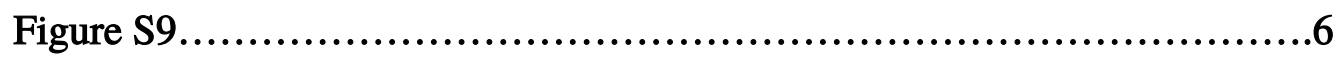

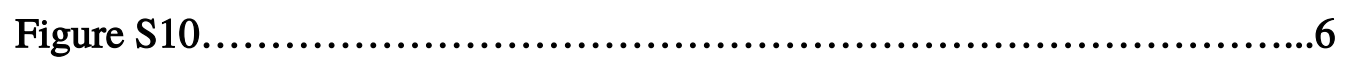




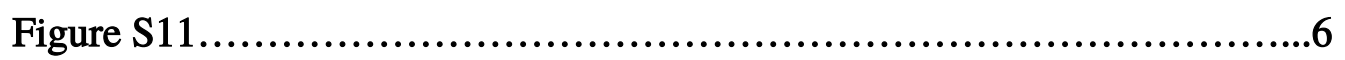

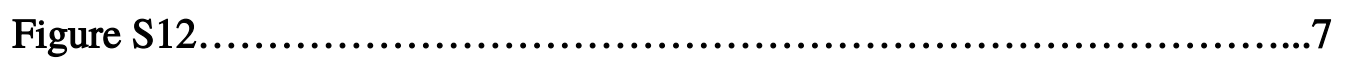

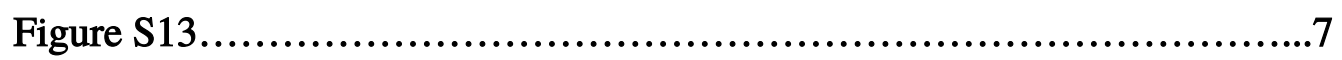

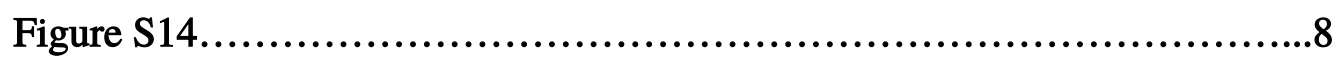

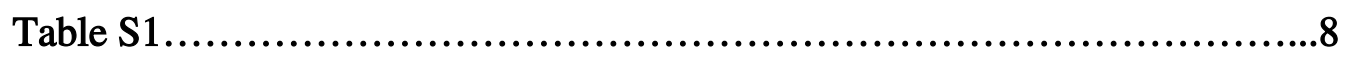

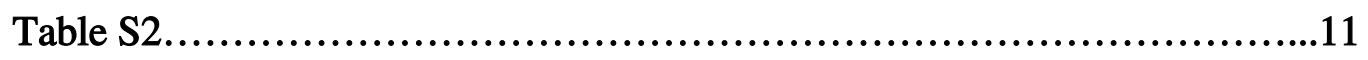

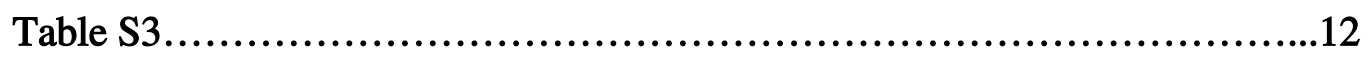

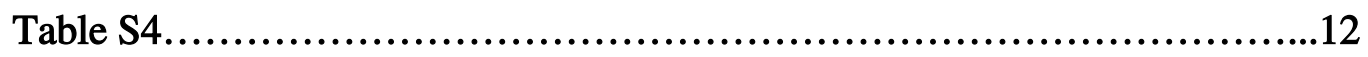

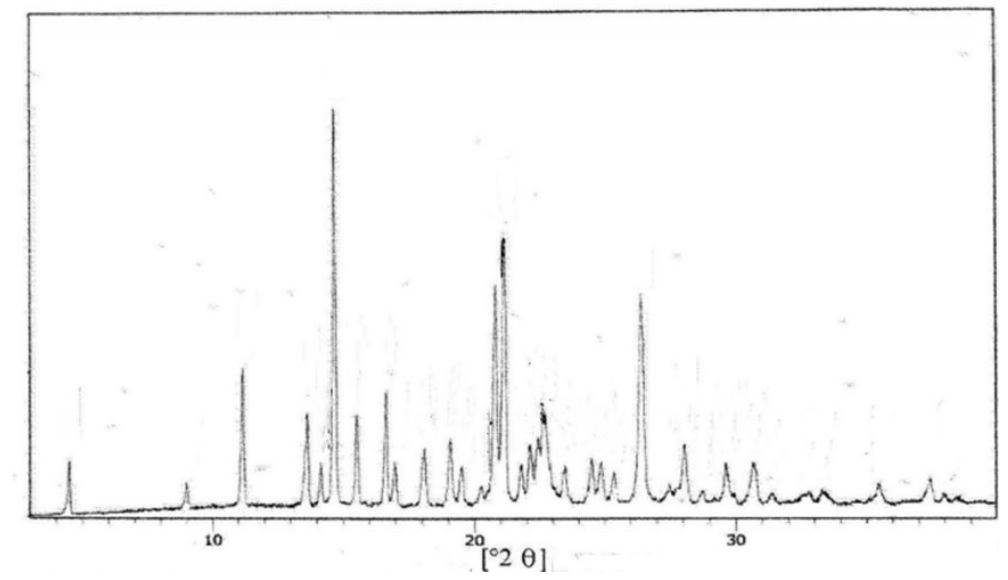

(a)

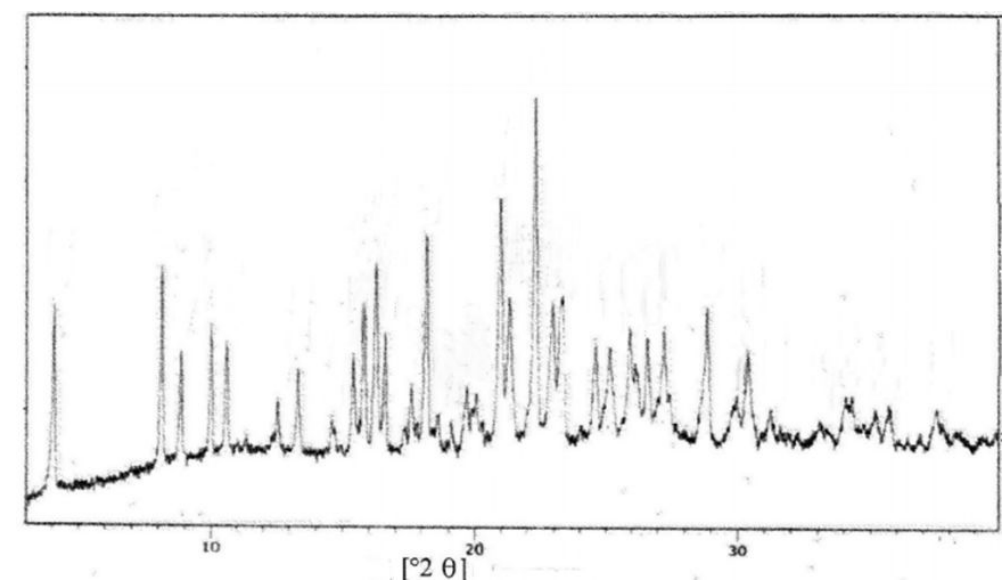

(b)

Figure S1. PXRD patterns provided in the reported patent ${ }^{1}$ (a) Form I, (b) belinostat acetone solvate. 


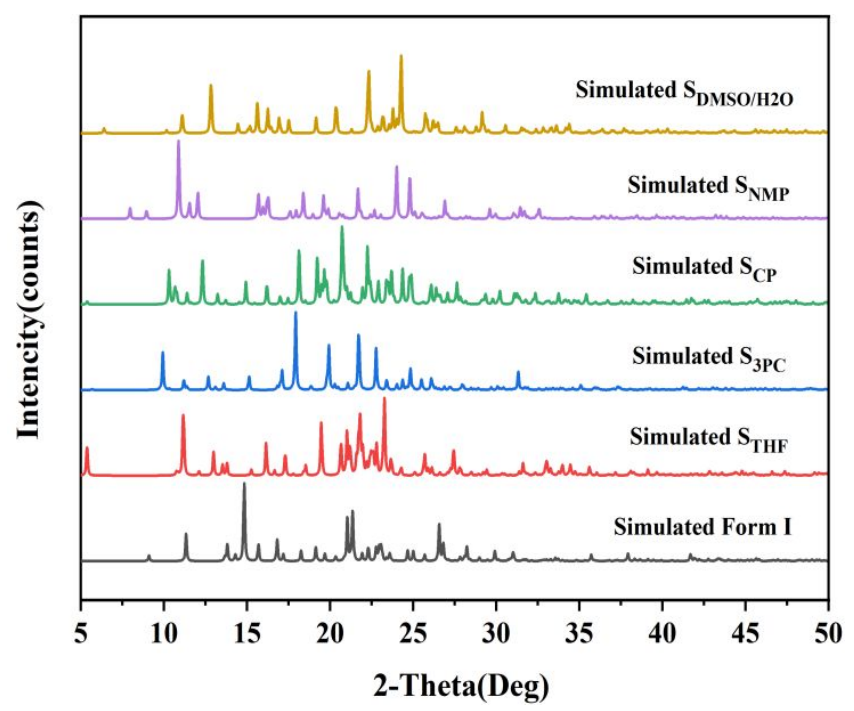

Figure S2. The simulated PXRD patterns of the solid forms of belinostat.

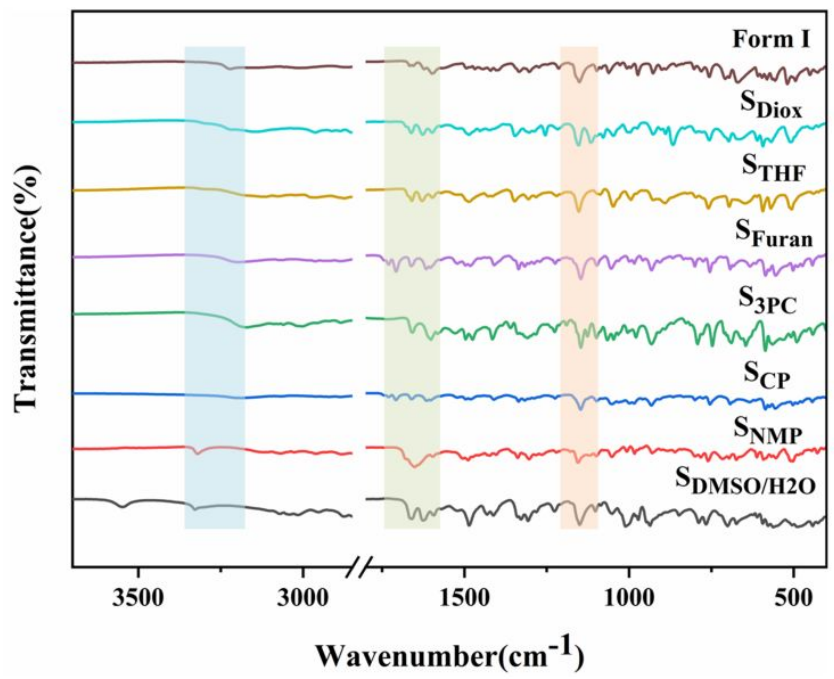

Figure S3. FT-IR spectra of the solid forms of belinostat. 


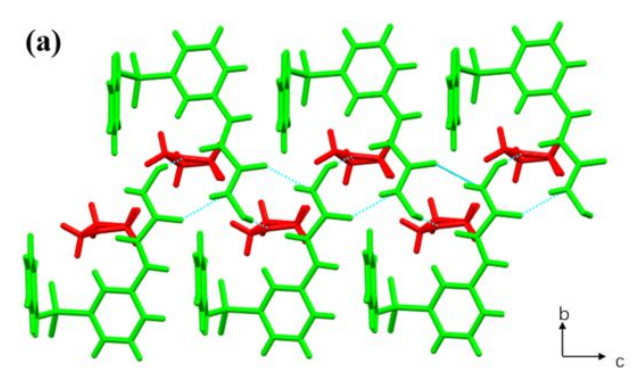

(b)
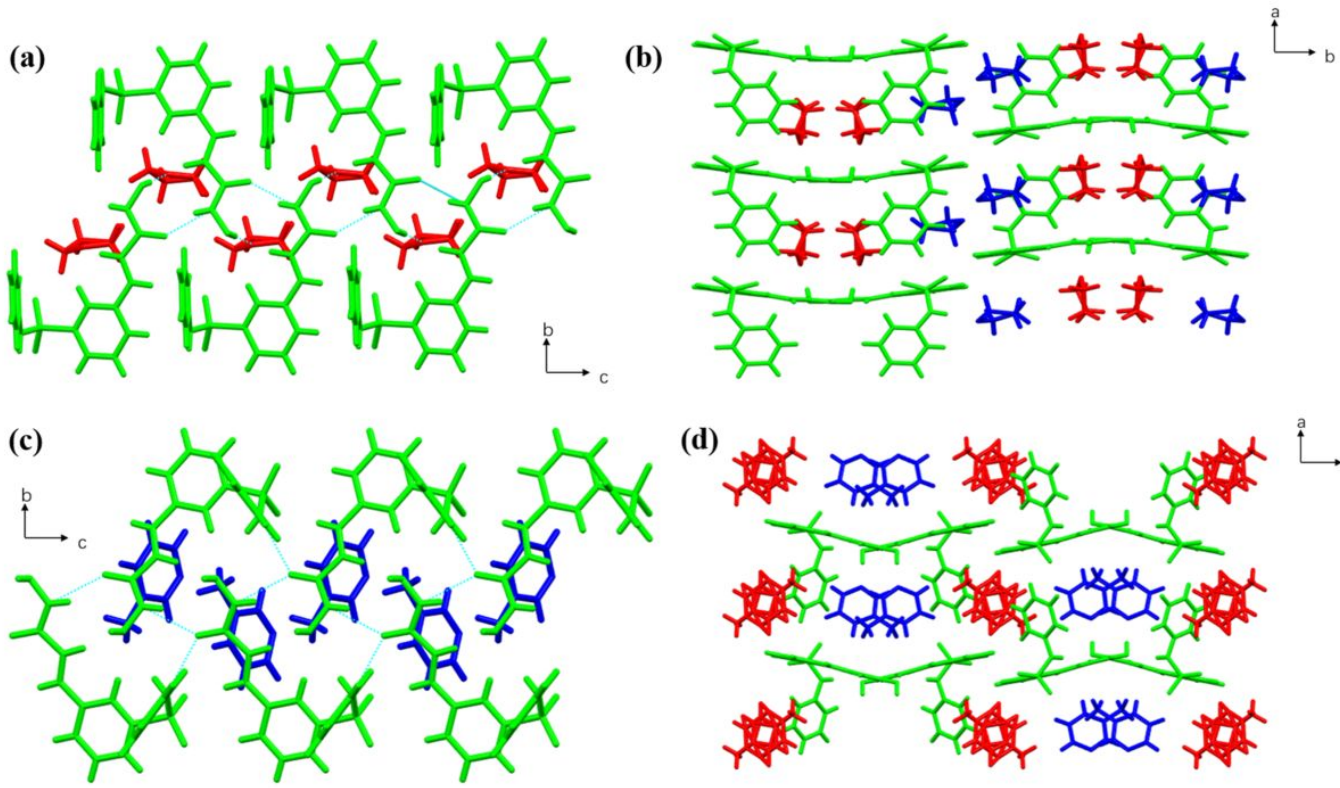

(d)
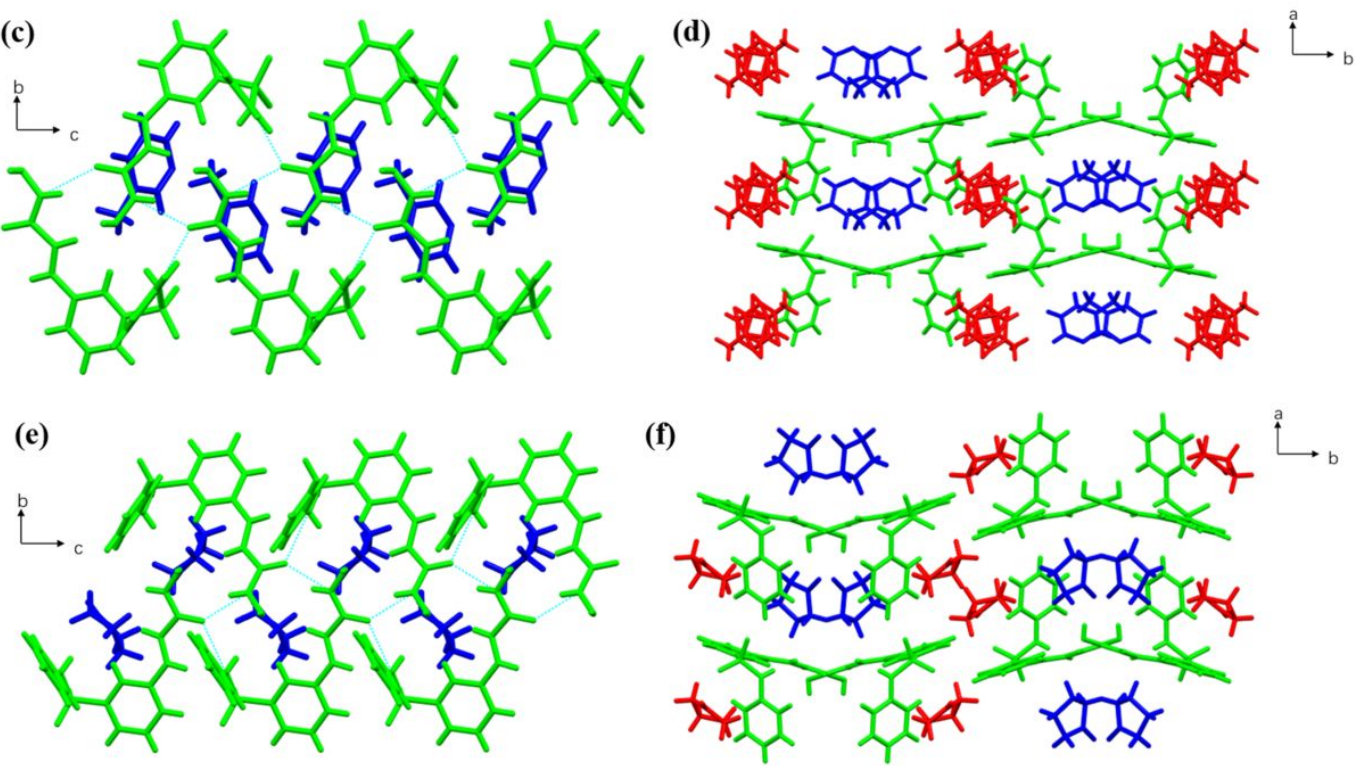

Figure S4. Comparison of crystal structures in the $b c$-plane, (a) corrugated 1D chain structure for $\mathrm{S}_{\mathrm{THF}}$, (c) for $\mathrm{S}_{3 \mathrm{PC}}$, (e) for $\mathrm{S}_{\mathrm{CP}}$; the intermolecular interactions present in the $a b$-plane (b) for $\mathrm{S}_{\mathrm{THF}}$, (d) for $\mathrm{S}_{3 \mathrm{PC},}(\mathrm{f})$ for $\mathrm{S}_{\mathrm{CP}}$.

(a)
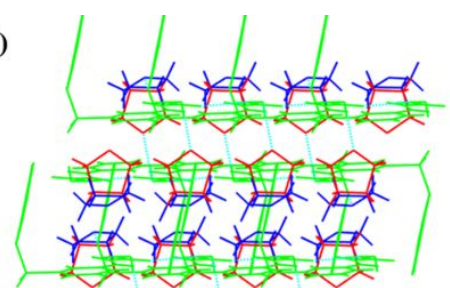

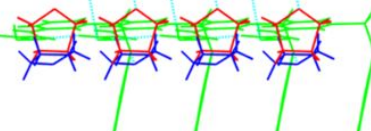

(b)
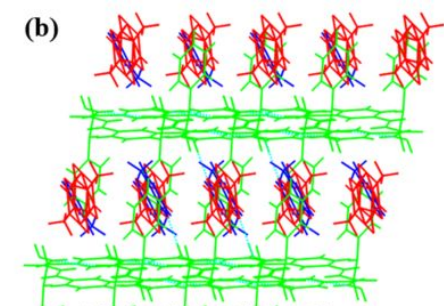

.
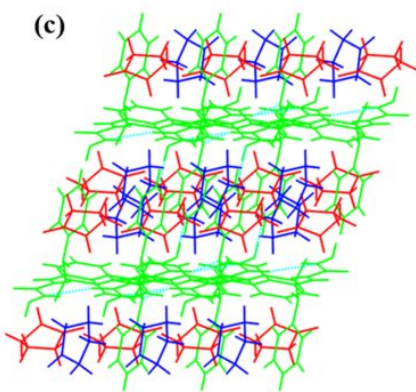

Figure S5. Comparison of crystal structures in $a c$-plane, (a) $\mathrm{S}_{\mathrm{THF}}$, (b) $\mathrm{S}_{3 \mathrm{PC}}$, (c) $\mathrm{S}_{\mathrm{CP}}$. 


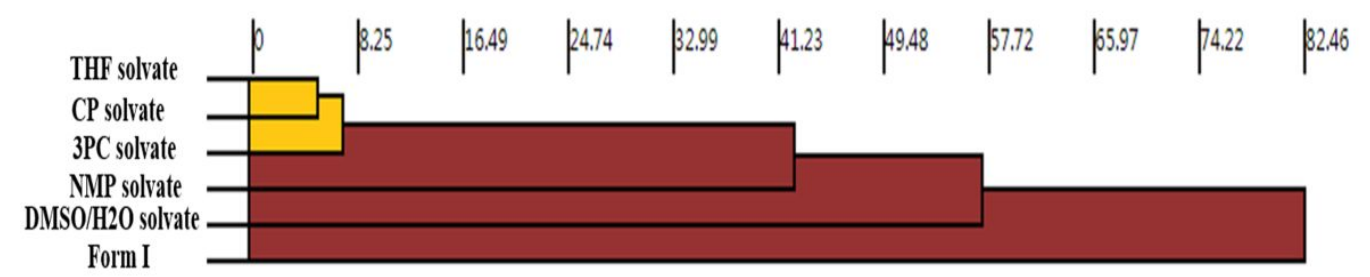

Figure S6. Packing similarity tree diagram of five solvates and Form I of belinostat calculated by CrystalCMP.

\section{Solid-State ${ }^{13} \mathrm{C}$ NMR}

${ }^{13} \mathrm{C}$ solid-state NMR spectra of $\mathrm{S}^{\text {Diox }}$ was obtained on a JEOL JNM ECZ600R 600 MHz NMR system equipped with a 4-mm double-resonance magic angle spinning (MAS) probe using a Ramp-CP cross-polarization technique. Other experimental parameters include contact time of $2 \mathrm{~ms} \mathrm{CP}$ time, recycle delay of $3 \mathrm{~s}$, spinning speed of $18 \mathrm{kHz}$, and $80 \mathrm{kHz}$ TPPM (two-phase pulse modulation) proton-decoupling during acquisition.

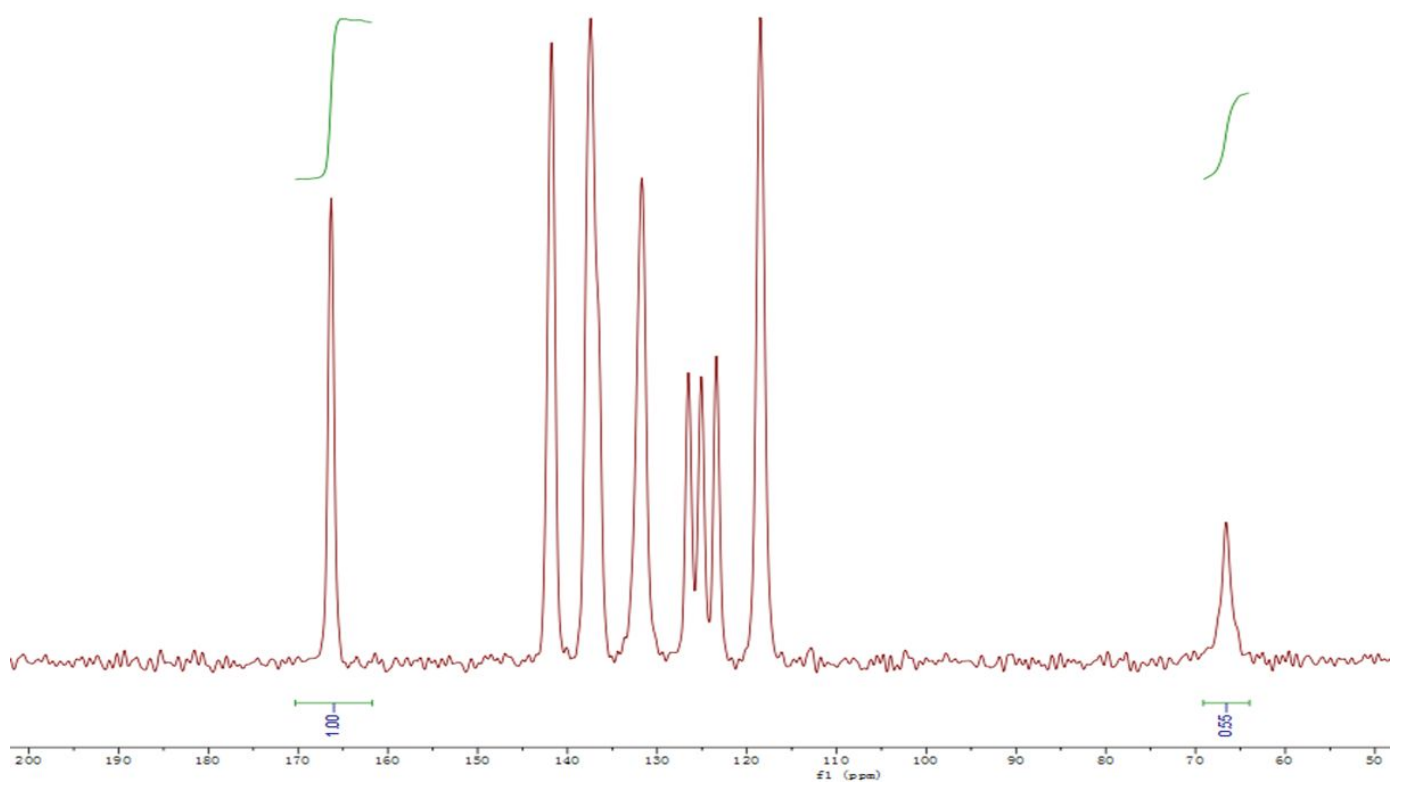

Figure S7. The ${ }^{13} \mathrm{C}-\mathrm{NMR}$ spectra for $\mathrm{S}_{\text {Diox }}$. 

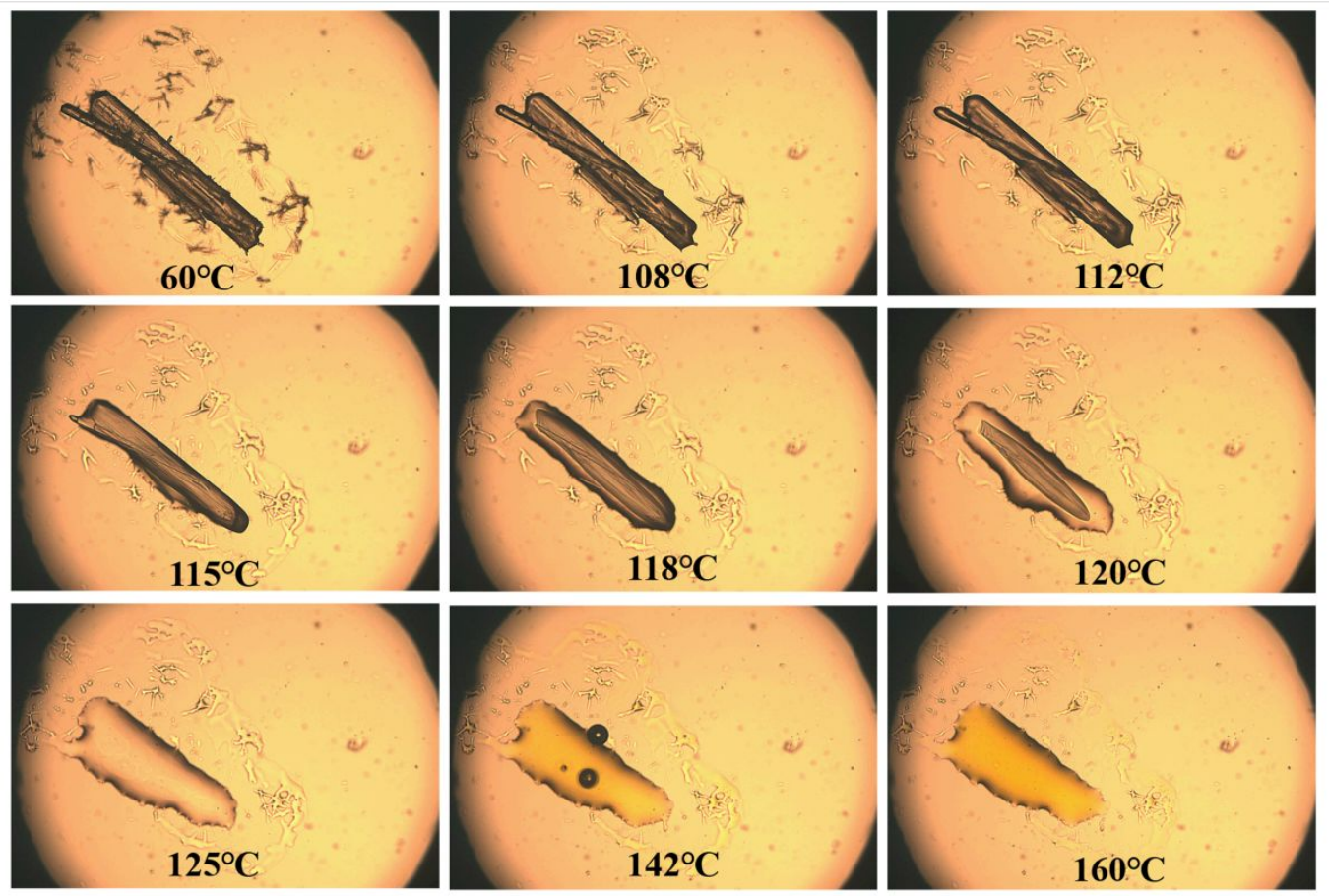

Figure S8. HSM images for belinostat solvate $S_{3 P C}$.
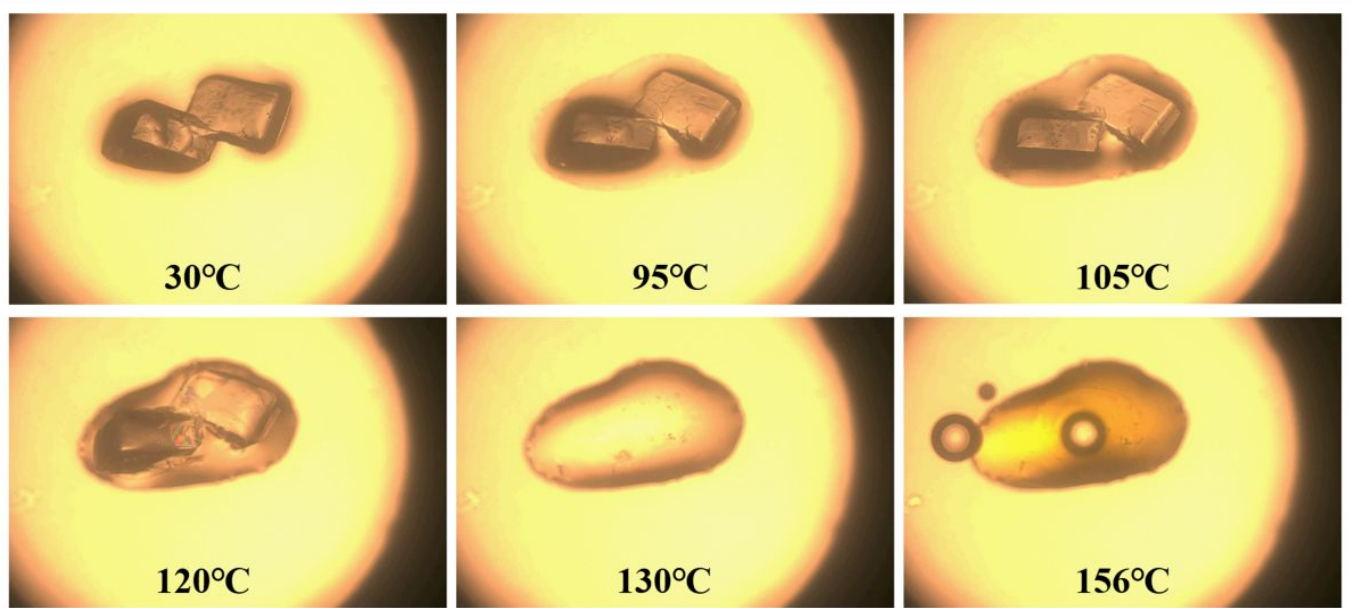

Figure S9. HSM images for belinostat solvate $\mathrm{S}_{\mathrm{NMP}}$. 


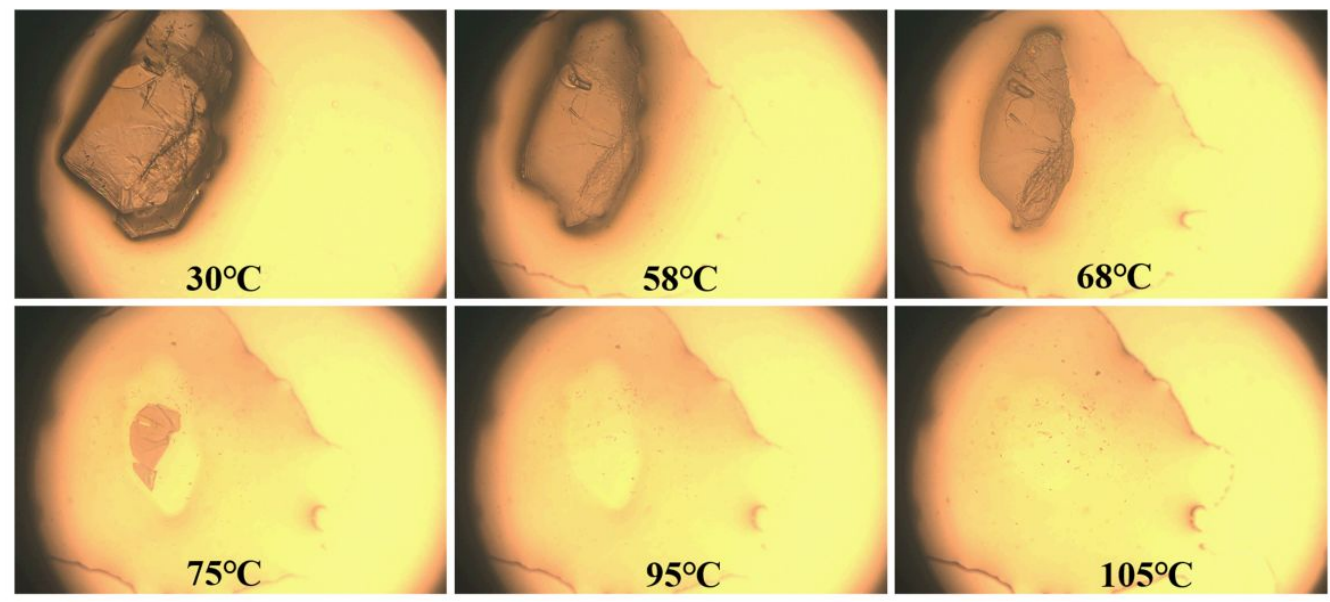

Figure S10. HSM images for belinostat solvate $\mathrm{S}_{\mathrm{DMSO} / \mathrm{H} 2 \mathrm{O}}$.

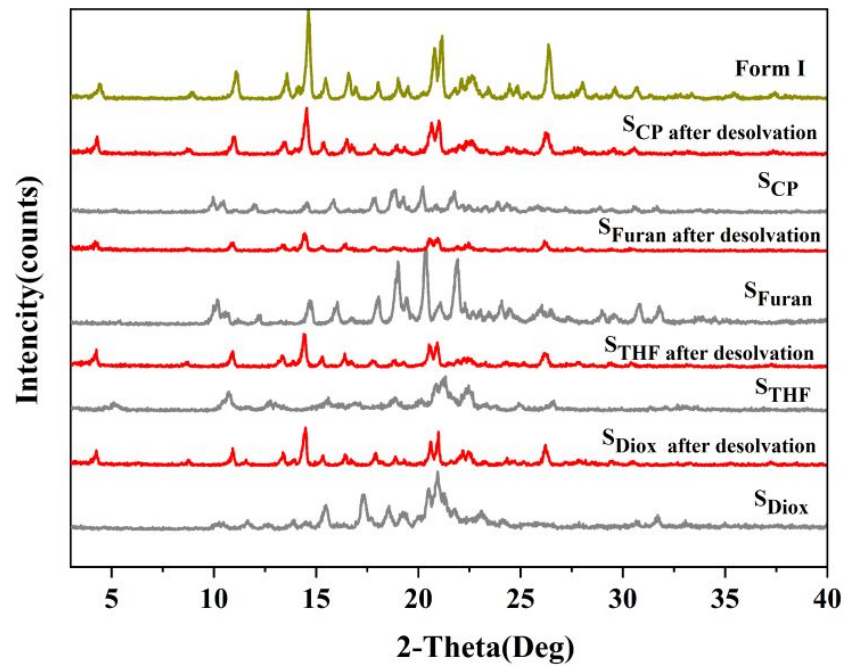

Figure S11. PXRD patterns of the desolvation products of $\mathrm{S}_{\mathrm{Diox}}, \mathrm{S}_{\mathrm{THF}}, \mathrm{S}_{\mathrm{Furan}}$ and $\mathrm{S}_{\mathrm{CP}}$.

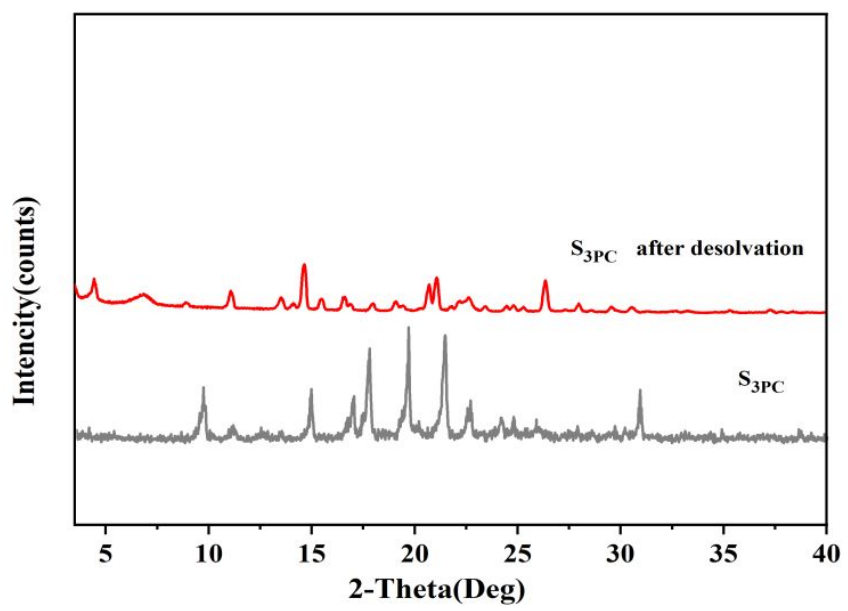

Figure S12. PXRD patterns of the desolvation products of $S_{3 p c}$. 


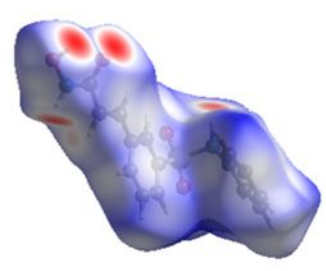

(a)

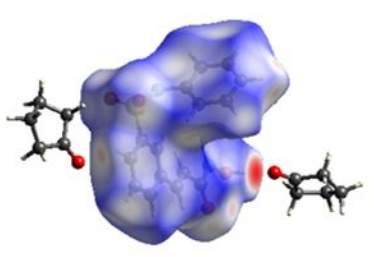

(d)

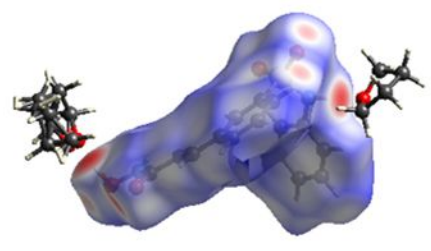

(b)

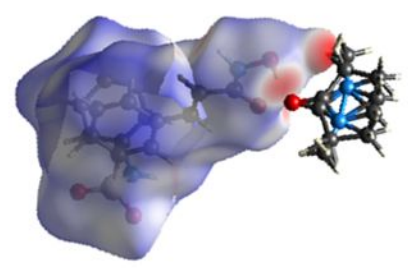

(e)

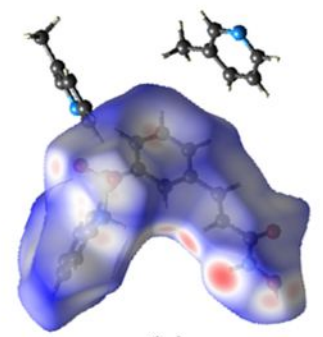

(c)

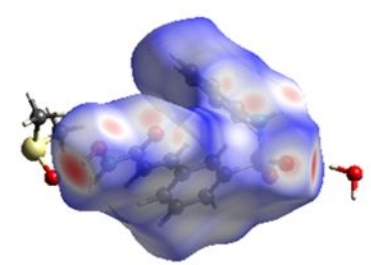

(f)

Figure S13. Hirshfeld surfaces of belinostat molecule in different solid forms for:

(a)Form I, (b) $\mathrm{S}_{\mathrm{THF}}$, (c) $\mathrm{S}_{3 \mathrm{PC}}$, (d) $\mathrm{S}_{\mathrm{CP}}$, (e) $\mathrm{S}_{\mathrm{NMP}}$, (f) $\mathrm{S}_{\mathrm{DMSO} / \mathrm{H} 2 \mathrm{O}}$.
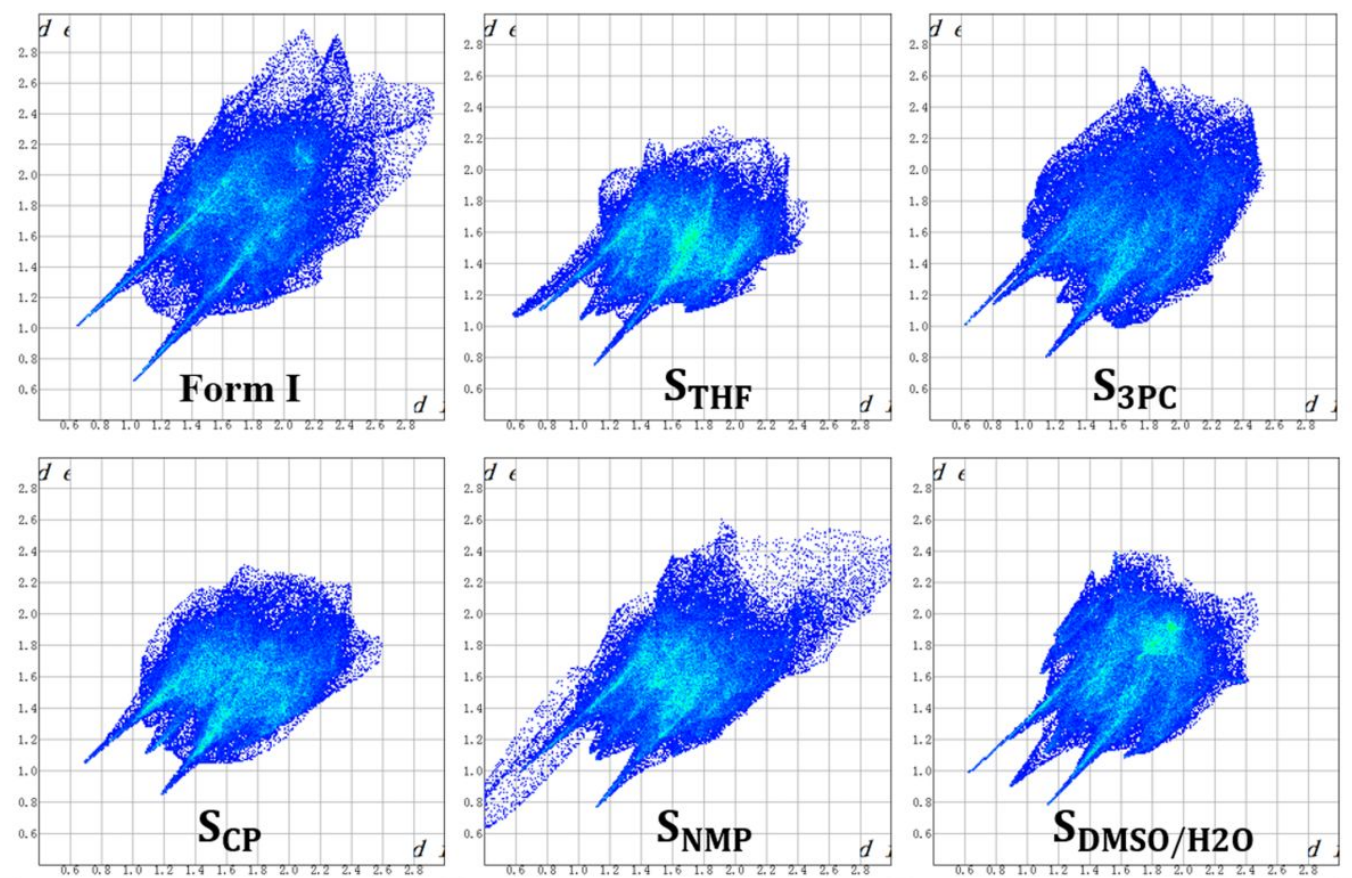

Figure S14. 2D fingerprint plots of belinostat molecule in different solid forms for:

(a)Form I, (b) $\mathrm{S}_{\mathrm{THF}}$, (c) $\mathrm{S}_{3 \mathrm{PC}}$, (d) $\mathrm{S}_{\mathrm{CP}}$, (e) $\mathrm{S}_{\mathrm{NMP}}$, (f) $\mathrm{S}_{\mathrm{DMSO} / \mathrm{H} 2 \mathrm{O}}$. 
Table S1. Summary of belinostat solid forms screening experiments.

\begin{tabular}{|c|c|c|c|c|c|}
\hline $\begin{array}{l}\text { Solvent } \\
\text { type }\end{array}$ & $\begin{array}{c}\text { Solvent } \\
\text { (//Antisolvent) }\end{array}$ & $\begin{array}{l}\text { Screening } \\
\text { method }\end{array}$ & $\begin{array}{l}\text { Starting } \\
\text { Material }\end{array}$ & $\begin{array}{c}\text { Temperature } \\
\left({ }^{\circ} \mathrm{C}\right)\end{array}$ & Solid Form \\
\hline \multirow{30}{*}{ H-bond acceptor } & N,N-Dimethylacetamide & Slurry & Form I & 30 & Form I \\
\hline & NMP & Slurry & Form I & 30 & Form I \\
\hline & DMSO & Slurry & Form I & 30 & Form I \\
\hline & $3 \mathrm{PC}$ & Slurry & Form I & 50 & $\mathrm{~S}_{3 \mathrm{PC}}$ \\
\hline & 1,4-Diox & Slurry & Form I & 50 & $\mathrm{~S}_{\text {Diox }}$ \\
\hline & THF & Slurry & Form I & 50 & $\mathrm{~S}_{\mathrm{THF}}$ \\
\hline & acetone & Slurry & Form I & 50 & Form I \\
\hline & $\mathrm{CP}$ & Slurry & Form I & 50 & $\mathrm{~S}_{\mathrm{CP}}$ \\
\hline & N,N-Dimethylformamide & Slurry & Form I & 30 & Form I \\
\hline & furan & Slurry & Form I & 50 & $\mathrm{~S}_{\text {Furan }}$ \\
\hline & N,N-Dimethylacetamide & cooling & Form I & $50-25$ & Form I \\
\hline & NMP & cooling & Form I & $50-25$ & Form I \\
\hline & DMSO & cooling & Form I & $50-25$ & Form I \\
\hline & $3 \mathrm{PC}$ & cooling & Form I & $50-25$ & $\mathrm{~S}_{3 \mathrm{PC}}$ \\
\hline & 1,4-Diox & cooling & Form I & $50-25$ & $\mathrm{~S}_{\text {Diox }}$ \\
\hline & THF & cooling & Form I & $50-25$ & $\mathrm{~S}_{\mathrm{THF}}$ \\
\hline & acetone & cooling & Form I & $50-25$ & Form I \\
\hline & $\mathrm{CP}$ & cooling & Form I & $50-25$ & $\mathrm{~S}_{\mathrm{CP}}$ \\
\hline & N,N-Dimethylformamide & cooling & Form I & $50-25$ & Form I \\
\hline & furan & cooling & Form I & $50-25$ & $\mathrm{~S}_{\text {Furan }}$ \\
\hline & N,N-Dimethylacetamide & evaporation & Form I & 30 & Form I \\
\hline & NMP & evaporation & Form I & 30 & Form I \\
\hline & DMSO & evaporation & Form I & 30 & Form I \\
\hline & acetone & evaporation & Form I & 30 & Form I \\
\hline & N,N-Dimethylformamide & evaporation & Form I & 30 & Form I \\
\hline & $\begin{array}{c}\text { N,N-Dimethylacetamide } \\
\text { //water }\end{array}$ & $\begin{array}{l}\text { Antisolvent } \\
\text { Addition }\end{array}$ & Form I & 30 & Form I \\
\hline & $\mathrm{NMP} / /$ water & $\begin{array}{l}\text { Antisolvent } \\
\text { Addition }\end{array}$ & Form I & 30 & Form I \\
\hline & DMSO//water & $\begin{array}{c}\text { Antisolvent } \\
\text { Addition }\end{array}$ & Form I & 30 & Form I \\
\hline & Acetone//water & $\begin{array}{c}\text { Antisolvent } \\
\text { Addition }\end{array}$ & Form I & 30 & Form I \\
\hline & N,N-Dimethylformamide & Antisolvent & Form I & 30 & Form I \\
\hline
\end{tabular}




\begin{tabular}{|c|c|c|c|c|c|}
\hline & //water & Addition & & & \\
\hline & $\begin{array}{c}\text { N,N-Dimethylacetamide } \\
+ \text { water }\end{array}$ & evaporation & Form I & RT & Form I \\
\hline & NMP+water & evaporation & Form I & RT & $\mathrm{S}_{\mathrm{NMP}}$ \\
\hline & DMSO+water & evaporation & Form I & RT & $\mathrm{S}_{\mathrm{DMSO} / \mathrm{H} 2 \mathrm{O}}$ \\
\hline & Acetone+water & evaporation & Form I & RT & Form I \\
\hline & $\begin{array}{c}\text { N,N-Dimethylformamide } \\
+ \text { +water }\end{array}$ & evaporation & Form I & RT & Form I \\
\hline \multirow{29}{*}{ H-bond donor } & formamide & Slurry & Form I & 30 & Form I \\
\hline & isopropanol & Slurry & Form I & 50 & Form I \\
\hline & ethanol & Slurry & Form I & 50 & Form I \\
\hline & isobutanol & Slurry & Form I & 50 & Form I \\
\hline & methanol & Slurry & Form I & 50 & Form I \\
\hline & 1-propanol & Slurry & Form I & 50 & Form I \\
\hline & 1-butanol & Slurry & Form I & 50 & Form I \\
\hline & pentanol & Slurry & Form I & 50 & Form I \\
\hline & ethylene glycol & Slurry & Form I & 50 & Form I \\
\hline & water & Slurry & Form I & 75 & Form I \\
\hline & formamide & evaporation & Form I & 30 & Form I \\
\hline & isopropanol & evaporation & Form I & 30 & Form I \\
\hline & ethanol & evaporation & Form I & 30 & Form I \\
\hline & isobutanol & evaporation & Form I & 30 & Form I \\
\hline & methanol & evaporation & Form I & 30 & Form I \\
\hline & 1-propanol & evaporation & Form I & 30 & Form I \\
\hline & 1-butanol & evaporation & Form I & 30 & Form I \\
\hline & pentanol & evaporation & Form I & 30 & Form I \\
\hline & ethylene glycol & evaporation & Form I & 30 & Form I \\
\hline & water & evaporation & Form I & 30 & Form I \\
\hline & formamide & cooling & Form I & $50-25$ & Form I \\
\hline & isopropanol & cooling & Form I & $50-25$ & Form I \\
\hline & ethanol & cooling & Form I & $50-25$ & Form I \\
\hline & isobutanol & cooling & Form I & $50-25$ & Form I \\
\hline & methanol & cooling & Form I & $50-25$ & Form I \\
\hline & 1-propanol & cooling & Form I & $50-25$ & Form I \\
\hline & 1-butanol & cooling & Form I & $50-25$ & Form I \\
\hline & pentanol & cooling & Form I & $50-25$ & Form I \\
\hline & ethylene glycol & cooling & Form I & $50-25$ & Form I \\
\hline
\end{tabular}




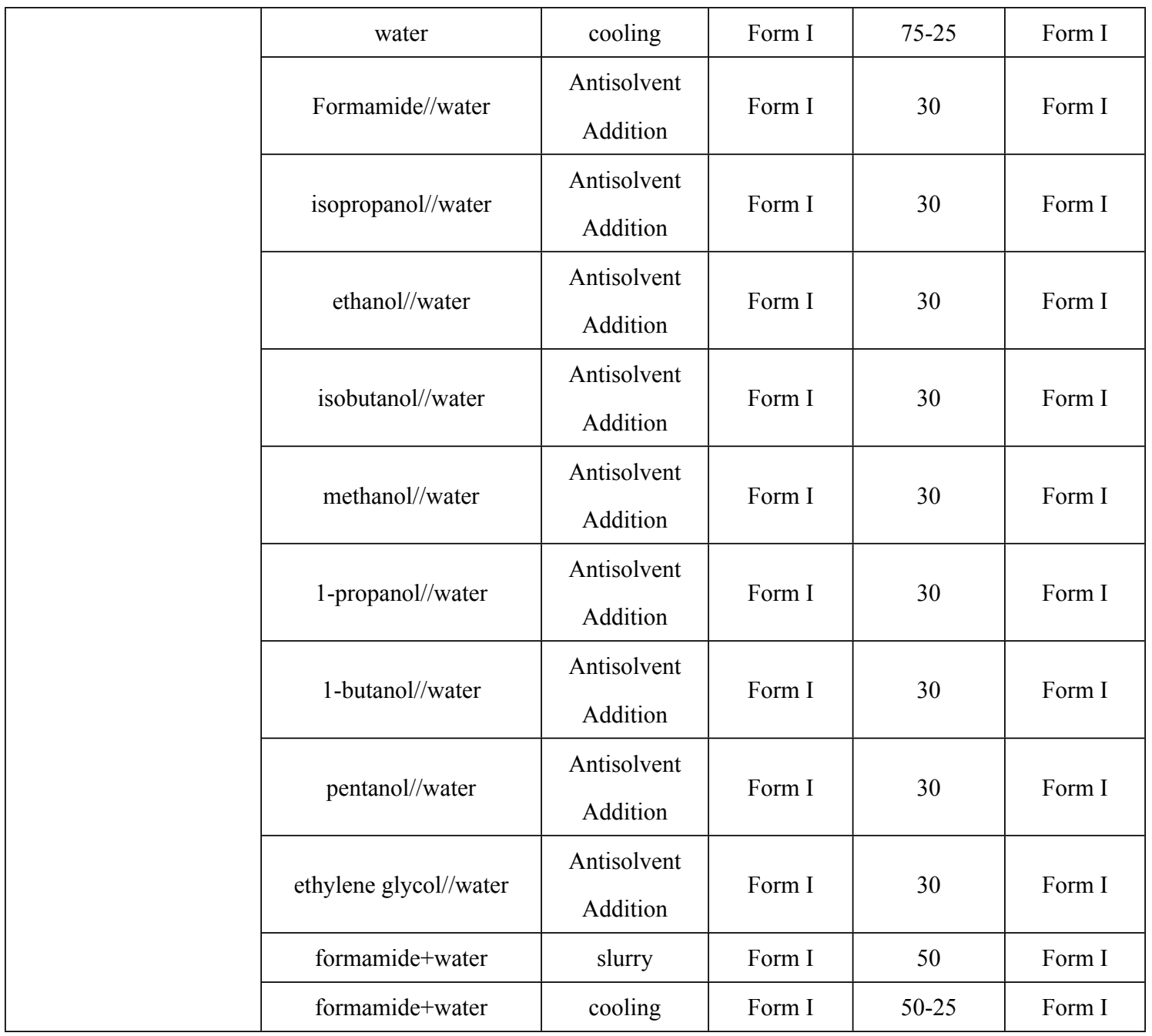

Table S2. Hydrogen bonds in crystal structures of solid forms of belinostat.

\begin{tabular}{|c|c|c|c|c|c|c|}
\hline & $\mathrm{D}-\mathrm{H} \cdots \mathrm{A}$ & $\mathrm{d}(\mathrm{D}-\mathrm{H}), \quad \AA$ & $\mathrm{d}(\mathrm{H} \cdots \mathrm{A}), \AA$ & $\mathrm{d}(\mathrm{D}-\mathrm{A}), \quad \AA$ & $\begin{array}{c}\angle \text { (DHA) } \\
\text { deg. }\end{array}$ & symmetric code \\
\hline \multirow[t]{4}{*}{ Form I } & $\mathrm{C} 14-\mathrm{H} 14 \cdots \mathrm{O} 3$ & $0.9501(14)$ & $2.477(11)$ & $3.2192(17)$ & $134.873(82)$ & $x-1 / 2,-y+3 / 2,-z+1$ \\
\hline & $\mathrm{N} 1-\mathrm{H} 1 \cdots \mathrm{O} 2$ & $0.839(19)$ & $2.151(18)$ & $2.9832(16)$ & $171.682(1689)$ & $-x+3 / 2, y+1 / 2, z$ \\
\hline & $\mathrm{O} 4-\mathrm{H} 4 \cdots \mathrm{O} 3$ & $0.88(2)$ & $1.7609(187)$ & $2.6240(16)$ & $168.036(1822)$ & $-\mathrm{x}+1,-\mathrm{y}+2,-\mathrm{z}+1$ \\
\hline & $\mathrm{N} 2-\mathrm{H} 2 \cdots \mathrm{O} 3$ & $0.90(2)$ & $1.8565(174)$ & $2.7360(16)$ & $164.398(1625)$ & $x-1 / 2,-y+3 / 2,-z+1$ \\
\hline \multirow[t]{8}{*}{$\mathrm{S}_{\mathrm{THF}}$} & $\mathrm{C} 2-\mathrm{H} 2 \mathrm{~A} \cdots \mathrm{O} 2$ & $0.9499(18)$ & $2.3840(12)$ & $3.0212(20)$ & $124.146(112)$ & \\
\hline & $\mathrm{C} 12-\mathrm{H} 12 \cdots \mathrm{O} 1$ & $0.9499(17)$ & $2.4174(13)$ & $3.2528(22)$ & $146.597(105)$ & $-\mathrm{x}+1,-\mathrm{y}+1,-\mathrm{z}+1$ \\
\hline & $\mathrm{C} 14-\mathrm{H} 14 \cdots \mathrm{O} 4$ & $0.9503(17)$ & $2.4193(14)$ & $3.3452(23)$ & $164.634(104)$ & $\mathrm{x},-\mathrm{y}+3 / 2, \mathrm{z}+1 / 2$ \\
\hline & $\mathrm{C} 19^{\wedge} \mathrm{a}-\mathrm{H} 19 \mathrm{~A}^{\wedge} \mathrm{a} \cdots \mathrm{O} 2$ & $1.1324(243)$ & $2.6554(11)$ & $3.3092(161)$ & $115.80(85)$ & $x,-y+3 / 2, z-1 / 2$ \\
\hline & $\mathrm{N} 1-\mathrm{H} 1 \cdots \mathrm{O} 6$ & $0.87(2)$ & $1.9912(230)$ & $2.8543(19)$ & $173.518(2197)$ & \\
\hline & $\mathrm{N} 2-\mathrm{H} 2 \cdots \mathrm{O} 3$ & $0.82(2)$ & $2.0231(265)$ & $2.8015(21)$ & $156.821(2364)$ & $\mathrm{x},-\mathrm{y}+3 / 2, \mathrm{z}+1 / 2$ \\
\hline & $\mathrm{O} 4-\mathrm{H} 4 \cdots \mathrm{O} 5^{\wedge} \mathrm{a}$ & $0.82(3)$ & $1.8273(231)$ & $2.6488(35)$ & $175.013(2649)$ & \\
\hline & $\mathrm{O} 4-\mathrm{H} 4 \cdots \mathrm{O} 5^{\wedge} \mathrm{b}$ & $0.82(3)$ & $1.8111(256)$ & $2.6208(103)$ & $167.279(2693)$ & \\
\hline \multirow[t]{2}{*}{$\mathrm{S}_{3 \mathrm{PC}}$} & $\mathrm{O} 4-\mathrm{H} 4 \cdots \mathrm{N} 3$ & $0.8400(14)$ & $1.7789(17)$ & $2.6181(22)$ & $176.968(128)$ & $-x, y-1 / 2,-z+1 / 2$ \\
\hline & $\mathrm{N} 1-\mathrm{H} 1 \cdots \mathrm{O} 4$ & $0.8602(17)$ & $2.5266(13)$ & $3.3790(21)$ & $171.064(110)$ & $x,-y+1 / 2, z+1 / 2$ \\
\hline
\end{tabular}




\begin{tabular}{|c|c|c|c|c|c|c|}
\hline & $\mathrm{N} 2-\mathrm{H} 2 \cdots \mathrm{O} 3$ & $0.8800(18)$ & $2.0567(14)$ & $2.8340(22)$ & $146.744(113)$ & $x,-y+1 / 2, z+1 / 2$ \\
\hline & $\mathrm{C} 12-\mathrm{H} 12 \cdots \mathrm{O} 2$ & $0.9501(22)$ & $2.5699(13)$ & $3.1364(23)$ & $118.496(117)$ & $-\mathrm{x}+1,-\mathrm{y}+1,-\mathrm{z}+2$ \\
\hline & $\mathrm{C} 8-\mathrm{H} 8 \cdots \mathrm{O} 4$ & $0.9503(19)$ & $2.4858(14)$ & $3.4082(23)$ & $163.676(116)$ & $x,-y+1 / 2, z+1 / 2$ \\
\hline & $\mathrm{C} 14-\mathrm{H} 14 \cdots \mathrm{O} 4$ & $0.9500(18)$ & $2.2977(17)$ & $3.2312(25)$ & 167.262(109) & $x,-y+1 / 2, z+1 / 2$ \\
\hline \multirow[t]{7}{*}{$\mathrm{S}_{\mathrm{CP}}$} & $\mathrm{O} 4-\mathrm{H} 4 \cdots \mathrm{O} 5$ & $0.8403(19)$ & $1.8804(27)$ & $2.7043(33)$ & $166.368(159)$ & \\
\hline & $\mathrm{N} 2-\mathrm{H} 2 \cdots \mathrm{O} 3$ & $0.8800(27)$ & $2.1425(20)$ & $2.9253(34)$ & $147.851(166)$ & $x,-y+3 / 2, z+1 / 2$ \\
\hline & $\mathrm{C} 2-\mathrm{H} 2 \mathrm{~A} \cdots \mathrm{O} 3$ & $0.9502(33)$ & $2.5413(19)$ & $3.3294(37)$ & $140.452(187)$ & $\mathrm{x}, \mathrm{y}, \mathrm{z}+1$ \\
\hline & $\mathrm{C} 6-\mathrm{H} 6 \cdots \mathrm{O} 6$ & $0.9498(28)$ & $2.5732(25)$ & $3.3787(40)$ & 142.771(191) & $-\mathrm{x}+1,-\mathrm{y}+1,-\mathrm{z}+1$ \\
\hline & $\mathrm{C} 8-\mathrm{H} 8 \cdots \mathrm{O} 4$ & $0.9499(25)$ & $2.6160(19)$ & $3.5583(32)$ & $171.561(163)$ & $x,-y+3 / 2, z+1 / 2$ \\
\hline & $\mathrm{C} 12-\mathrm{H} 12 \cdots \mathrm{O} 6$ & $0.9500(33)$ & $2.4624(26)$ & $3.2537(41)$ & $140.716(190)$ & \\
\hline & $\mathrm{C} 14-\mathrm{H} 14 \cdots \mathrm{O} 4$ & $0.9501(28)$ & $2.4203(16)$ & $3.263(3)$ & $147.695(151)$ & $x,-y+3 / 2, z+1 / 2$ \\
\hline \multirow[t]{6}{*}{$\mathrm{S}_{\mathrm{NMP}}$} & $\mathrm{C} 2-\mathrm{H} 2 \mathrm{~A} \cdots \mathrm{O} 2$ & $0.9500(18)$ & $2.5485(10)$ & $3.0807(17)$ & 115.611(93) & \\
\hline & $\mathrm{C} 20 \mathrm{~A}^{\wedge} \mathrm{b}-\mathrm{H} 20 \mathrm{D}^{\wedge} \mathrm{b} \cdots \mathrm{O} 4$ & $0.9809(133)$ & $2.4654(13)$ & $3.3892(140)$ & $156.831(634)$ & \\
\hline & $\mathrm{N} 1-\mathrm{H} 1 \cdots \mathrm{O} 4$ & $0.859(18)$ & $2.6575(152)$ & $3.2466(13)$ & $126.91(1478)$ & $-\mathrm{x}+1,-\mathrm{y}+1,-\mathrm{z}+1$ \\
\hline & $\mathrm{N} 1-\mathrm{H} 1 \cdots \mathrm{O} 3$ & $0.859(18)$ & $2.0265(211)$ & $2.8607(17)$ & $163.662(1674)$ & $-\mathrm{x}+1,-\mathrm{y}+1,-\mathrm{z}+1$ \\
\hline & $\mathrm{N} 2-\mathrm{H} 2 \cdots \mathrm{O} 1$ & $0.868(17)$ & $2.1058(143)$ & $2.9530(13)$ & $165.088(1600)$ & $\mathrm{x}+1, \mathrm{y}, \mathrm{z}$ \\
\hline & $\mathrm{O} 4-\mathrm{H} 4 \cdots \mathrm{O} 5$ & $0.88(2)$ & $1.7259(193)$ & $2.5972(13)$ & $174.615(1951)$ & \\
\hline \multirow[t]{7}{*}{$\mathrm{S}_{\mathrm{DMSO} / \mathrm{H} 2 \mathrm{O}}$} & $\mathrm{O} 4-\mathrm{H} 4 \cdots \mathrm{O} 6$ & $0.8399(15)$ & $1.758(16)$ & $2.594(22)$ & $173.48(10)$ & $\mathrm{x}, \mathrm{y}, \mathrm{z}+1$ \\
\hline & $\mathrm{N} 1-\mathrm{H} 1 \cdots \mathrm{O} 4$ & $0.8600(15)$ & $2.4020(14)$ & $3.0078(20)$ & $127.892(107)$ & $-\mathrm{x},-\mathrm{y}+1,-\mathrm{z}+1$ \\
\hline & $\mathrm{N} 2-\mathrm{H} 2 \cdots \mathrm{O} 5$ & $0.8797(15)$ & $2.1595(14)$ & $2.8238(20)$ & 131.892(113) & \\
\hline & $\mathrm{C} 2-\mathrm{H} 2 \mathrm{~A} \cdots \mathrm{O} 1$ & $0.9501(18)$ & $2.5437(14)$ & $3.0718(22)$ & $115.267(107)$ & \\
\hline & $\mathrm{C} 6-\mathrm{H} 6 \cdots \mathrm{O} 3$ & $0.9504(18)$ & $2.5495(13)$ & $3.2922(22)$ & 135.151(111) & $-\mathrm{x},-\mathrm{y}+1,-\mathrm{z}+1$ \\
\hline & $\mathrm{C} 8-\mathrm{H} 8 \cdots \mathrm{O} 3$ & $0.9502(16)$ & $2.6141(13)$ & $3.4267(21)$ & 143.741(102) & $-\mathrm{x},-\mathrm{y}+1,-\mathrm{z}+1$ \\
\hline & $\mathrm{C} 16-\mathrm{H} 16 \mathrm{C} \cdots \mathrm{O} 1$ & $0.9798(24)$ & $2.331(13)$ & $3.2107(28)$ & $148.990(151)$ & $-\mathrm{x}+1,-\mathrm{y},-\mathrm{z}+1$ \\
\hline
\end{tabular}

Table S3. Torsion angles $\left(\tau,{ }^{\circ}\right)$ in belinostat Form I and its solvates.

\begin{tabular}{|c|c|c|c|c|c|c|}
\hline Torsion Angle & Form I & $\mathrm{S}_{\mathrm{THF}}$ & $\mathrm{S}_{3 \mathrm{PC}}$ & $\mathrm{S}_{\mathrm{CP}}$ & $\mathrm{S}_{\mathrm{NMP}}$ & $\mathrm{S}_{\mathrm{DMSO} / \mathrm{H} 2 \mathrm{O}}$ \\
\hline$\tau(\mathrm{C} 7-\mathrm{S} 1-\mathrm{N} 1-\mathrm{C} 1)$ & $51.64(0.11)$ & $63.73(0.15)$ & $-78.27(0.17)$ & $-73.31(0.24)$ & $-52.43(0.11)$ & $-61.74(0.15)$ \\
\hline$\tau(\mathrm{S} 1-\mathrm{N} 1-\mathrm{C} 1-\mathrm{C} 6)$ & $-101.40(0.13)$ & $-140.26(0.14)$ & $-148.51(0.20)$ & $16.89(0.38)$ & $118.75(0.12)$ & $122.20(0.16)$ \\
\hline$\tau(\mathrm{C} 9-\mathrm{C} 13-\mathrm{C} 14-\mathrm{C} 15)$ & $1.11(0.13)$ & $-175.65(0.15)$ & 177.58 ( 0.19) & $176.04(0.26)$ & $2.46(0.11)$ & $179.58(0.16)$ \\
\hline$\tau(\mathrm{C} 8-\mathrm{C} 9-\mathrm{C} 13-\mathrm{C} 14)$ & $17.8(0.14)$ & $7.61(0.27)$ & $3.26(0.35)$ & $-15.47(0.44)$ & $7.62(0.12)$ & $-179.15(0.18)$ \\
\hline$\tau(\mathrm{C} 13-\mathrm{C} 14-\mathrm{C} 15-\mathrm{N} 2)$ & $8.63(0.13)$ & $173.47(0.16)$ & $176.63(0.21)$ & $-168.77(0.26)$ & $6.58(0.11)$ & $-178.75(0.17)$ \\
\hline$\tau(\mathrm{O} 4-\mathrm{N} 2-\mathrm{C} 15-\mathrm{C} 14)$ & $-172.39(0.12)$ & $-178.34(0.14)$ & $-175.62(0.16)$ & $180.00(0.22)$ & $-169.70(0.10)$ & $-173.87(0.15)$ \\
\hline
\end{tabular}

Table S4. The thermal stability analysis of belinostat solvates: volume of solvent 
molecules $(V)$, solvent $\mathrm{Mw}$, solvent boiling point, volume proportion of cavities (containing solvent molecules) and crystal lattice energy.

\begin{tabular}{cccccc}
\hline solid form & $V\left(\AA^{3}\right)$ & $\begin{array}{c}\text { solvent } \\
\mathrm{Mw}\end{array}$ & $\begin{array}{c}\text { boiling point } \\
\text { of solvent } /{ }^{\circ} \mathrm{C}\end{array}$ & $\begin{array}{c}\text { volume proportion } \\
\text { of cavities }\end{array}$ & $\begin{array}{c}\text { crystal lattice } \\
\text { energy }\left(\mathrm{kJ} \cdot \mathrm{mol}^{-1}\right)\end{array}$ \\
\hline $\mathrm{S}_{\mathrm{THF}}$ & 77.98 & 72.11 & 66 & $30.4 \%$ & -70.42 \\
$\mathrm{~S}_{3 \mathrm{PC}}$ & 96.45 & 93.13 & 144 & $44 \%$ & -78.84 \\
$\mathrm{~S}_{\mathrm{CP}}$ & 87.98 & 84.12 & 130 & $45.2 \%$ & -76.35 \\
$\mathrm{~S}_{\mathrm{NMP}}$ & 100.53 & 99.13 & 202 & $30.8 \%$ & -69.36 \\
$\mathrm{~S}_{\mathrm{DMSO} / \mathrm{H} 2 \mathrm{O}}$ & $71.43 / 19.33$ & $78.13 / 18$ & $189 / 100$ & $27.2 \%$ & -68.38 \\
\hline
\end{tabular}

\section{REFERENCES}

(1) Male, S. R.; Upadhyay, S.; Sharma, S. K.; Acharya, H.; Singh, G.; Lahiri, S.;

Cabri, W. CN 109496210 A, 2017. 